\title{
Lumbar spine bone mineral density and trabecular bone score-adjusted FRAX, but not FRAX without bone mineral density, identify subclinical carotid atherosclerosis
}

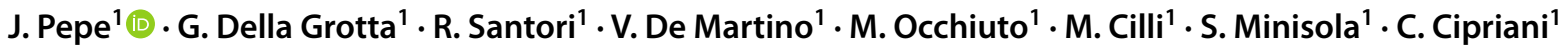

Received: 2 January 2021 / Accepted: 20 January 2021 / Published online: 12 February 2021

(C) The Author(s) 2021

\begin{abstract}
Purpose Osteoporosis and atherosclerosis share common risk factors. Aim of this study was to test if FRAX (which is an algorithm that can identify subjects at risk of fracture), without or with BMD values, also adjusted for trabecular bone score (TBS) was able to identify subclinical atherosclerosis, evaluated by measurement of carotid intima media thickness (cIMT $\geq 0.9 \mathrm{~mm}$ ) as compared to DXA values.

Methods Ninety postmenopausal women underwent DXA measurement and cIMT evaluation. For each patient, the FRAX algorithm for major osteoporotic fracture $(\mathrm{M})$ and for hip fracture $(\mathrm{H})$ without BMD was computed, together with FRAX with BMD and TBS-adjusted FRAX. Serum levels of osteoprotegerin, sRANKL, and interleukin-6 were also measured.

Results There were no differences in anthropometric parameters and cardiovascular risk factors between subjects with cIMT $\geq 0.9 \mathrm{~mm}$ ( $35 \%$ of subjects, group A) compared to those with cIMT $<0.9 \mathrm{~mm}$ (group B). The prevalence of osteoporosis and FRAX BMD, TBS-adjusted FRAX both for $\mathrm{M}$ and $\mathrm{H}$ were higher in group A compared to group $\mathrm{B}$. The best ROC curves to identify subjects with a cIMT $\geq 0.9 \mathrm{~mm}$ were: lumbar spine T-score, with a threshold of $-2.5 \mathrm{SD}$ (area under the curve, AUC $0.64 ; p=0.02$ ) with a sensibility of $50 \%$ and a specificity of $76 \%$; TBS-adjusted FRAX $\mathrm{H}$ with a sensibility of $50 \%$ and a specificity of $72 \%$ (AUC $0.64 ; p=0.01$ with a threshold of $3 \%$ ). Interleukin-6 positively correlated with FRAX BMD $\mathrm{H}$ and $\mathrm{M}$.

Conclusions FRAX without BMD does not identify subclinical carotid atherosclerosis, while lumbar spine T-score and TBS-adjusted FRAX H similarly detected it with higher specificity for T-score.
\end{abstract}

Keywords Atherosclerosis · Osteoporosis $\cdot$ Carotid intima media thickness $\cdot$ Trabecular bone score $\cdot$ FRAX

\section{Introduction}

Recent meta-analyses showed that low bone mineral density (BMD) is independently associated with cardiovascular diseases $[1,2]$ and in particular with atherosclerosis [3]. However, there are many risk factors in common between these two conditions that need to be taken into account and can probably influence the strength of this association.

Preclinical atherosclerosis can be assessed by carotid intima media thickness (cIMT) measurement, a widely

J. Pepe
jessica.pepe@uniroma1.it

Department of Clinical, Internal, Anesthesiology and Cardiovascular Sciences, (SCIAC), "Sapienza"

University of Rome, Rome, Italy accepted marker of atherosclerosis and predictor of future vascular events [4]. Several studies reported significantly higher cIMT values in patients with osteoporosis compared to non-osteoporotic subjects, which were negatively correlated with BMD values [5-8].

To explain this association, a possible common pathophysiological mechanism underlying these diseases still needs to be found. However, both vascular calcification and bone metabolism share some bone derived proteins such as osteocalcin [9] and osteoprotegerin (OPG) [10, 11]. Moreover, low vitamin D levels have been linked to both cardiovascular disease and bone fragility $[12,13]$, also because it causes secondary hyperparathyroidism [14]. Finally, inflammation with high interleukin 6 level has been reported in both diseases $[15,16]$.

Although these molecular shared pathways are still areas of research and uncertainty, there are well-established 
clinical risk factors for osteoporosis and cardiovascular disease [17-20].These are age, sex and specific lifestyle habits, such as excessive alcohol intake, smoking, high body mass index (BMI) and glucocorticoid use [21]. Cardiovascular diseases and osteoporosis are both more frequent in some endocrinological or rheumatological diseases such as rheumatoid arthritis [21]. Also, bone fragility fractures have been associated with cardiovascular disease, in particular, with development of carotid atherosclerosis [22]. All of the above mentioned shared clinical risk factors regarding bone fragility and cardiovascular disease are the risk factors included in a computer-free algorithm, FRAX®, created to predict 10 -year fracture risk in subjects ranging from 40 to 90 years of age. This algorithm also included the possibility to add the femoral neck bone mineral density (BMD) value or neck T-score [23]. A recent improvement of this tool was the possibility of adjusting FRAX values with the trabecular bone score (TBS), which is an indirect measure of bone quality, derived from the dual-energy X-ray absorptiometry (DXA) scan image of the lumbar spine with a specific software [24]. TBS has been associated with the risk of fractures independently of BMD [24].

To the best of our knowledge only one study has been carried out, showing a positive correlation between FRAX without BMD and a surrogate of peripheral atherosclerosis (brachial artery IMT) in a general population of men and women [25]. There are no studies which aimed to evaluate the performance of FRAX with or without BMD, or also with TBS-adjusted FRAX to identify patients with subclinical carotid atherosclerosis compared to DXA values alone. Aim of this study was to evaluate if FRAX, with or without BMD and also TBS-adjusted FRAX were able to identify postmenopausal women with subclinical atherosclerosis, evaluated with cIMT compared to DXA values alone. Several biochemical molecules that may explain the physiopathological link between atherosclerosis and osteoporosis such as OPG, soluble receptor activator of nuclear factor kappa-B ligand (sRANKL), and interleukin-6,were also measured.

\section{Methods}

\section{Patients}

Between January 2017 and April 2020, 100 consecutive postmenopausal women were enrolled at the Bone Disease Unit of "Sapienza" University during their first visit for osteoporosis screening. The inclusion criteria for this study were age range from 50 to 85 years and being postmenopausal. Based on medical interviews, we excluded patients with neoplasia, presence of previous cardiovascular diseases and diabetes mellitus type 1 and 2 . We did not measure serum glucose. None of the patients enrolled had ever undergone any treatment which could interfere with mineral metabolism (steroids, diuretics, thyroid hormones, anticonvulsant drugs, lithium, etc.). Furthermore, no patients reported a history of known secondary osteoporosis. Another exclusion criterium was an estimated creatinine clearance $<60 \mathrm{ml} / \mathrm{min}$. We did not perform a lipid panel measurement; however, dyslipidemia was anamnestically evaluated on the basis of the latest blood examination (no later than 6 months) [26]. Hypertension was ascertained through blood pressure measurement and defined as systolic blood pressure $>140 \mathrm{mmHg}$ or diastolic blood pressure $>90 \mathrm{mmHg}$.

All subjects gave written informed consent and the study was approved by the local hospital's Research Ethics Committee.

\section{Laboratory investigations}

Mineral metabolism was evaluated by measuring: serum total calcium $(\mathrm{Ca})$, phosphorus $(\mathrm{P}), 25$-hydroxyvitamin $\mathrm{D}$ $[25(\mathrm{OH}) \mathrm{D}]$, parathyroid hormone $(\mathrm{PTH})$ and creatinine $(\mathrm{Cr})$, as previously described [27, 28]. Creatinine clearance was estimated by the formula of Cockcroft and Gault. We utilized the ELISA kits for the following measurements: bone isoenzyme of alkaline phosphatase [AC-20, IDS, UK, inter-assay coefficient of variation $(\mathrm{CV})$ was $4.5 \%$ and intra-assay $\mathrm{CV}$ $5.9 \%$ ], C-terminal telopeptide of collagen type I (AC-02F1, IDS, UK inter-assay CV 5\%, intra-assay CV 6\%), receptor activator of nuclear factor kappa-B ligand (sRANKL, Biovendor, Czech Republic, inter-assay CV 7.2\%, intra-assay CV 9\%), OPG (Biovendor, Czech Republic, inter-assay CV 4.9\%, intra-assay 7.1\%) and Interleukin-6 (R\&D System, Minneapolis, USA inter-assay CV 4\%, intra-assay CV 6.3\%).

\section{Radiological examination}

Each subject underwent standardized spine lateral radiographs to detect vertebral deformity, defined according to Genant's method. BMD of the lumbar spine (L1-L4), in the anterior-posterior projection and of the hip (femoral neck and total hip), was measured in each patient with DXA scan (Hologic QDR 4500, Hologic Inc., Waltham, MA, USA). Fractured vertebrae were excluded. The precision error of lumbar spine and total hip measurement was 1.3 and $1.7 \%$, respectively [29]. DXA results of lumbar and/ or neck site were classified according to the World Health Organization (WHO) diagnostic criteria for osteoporosis [normal (T-score $\geq 1 \mathrm{SD}$ ), osteopenic (T-score between -1 and $<-2.5 \mathrm{SD}$ ) and osteoporotic (T-score $\leq-2.5 . \mathrm{SD}$ )]. TBS was computed, after fractured vertebrae were excluded, in the same regions as those used for lumbar spine BMD using TBS iNsight (version 3.0.2.0, Med-Imaps, Switzerland), as the mean value of the individual measurement for vertebrae 
L1-L4. The coefficient of variation for TBS was $1.8 \%$ and did not vary among the measured vertebrae.

\section{cIMT}

The evaluation of cIMT was accessed using high-resolution B-mode ultrasound (Mindray, dc-80, $\mathrm{x}$-insight) according to the protocols of the American Association of Echocardiography [30]. Early carotid atherosclerosis was defined as a mean $\operatorname{cIMT} \geq 0.9 \mathrm{~mm}$.

\section{FRAX}

For each patient, the FRAX algorithm was computed for major osteoporotic fracture (M), i.e., clinical spine, forearm, hip and proximal humerus and for hip fracture alone $(\mathrm{H})$, without BMD [31]. FRAX is a computer-based algorithm that provides the 10-year probability of fractures in men and women on the basis of classic risk factors (CRFs), such as age, sex, weight, height, previous fracture, parent hip fracture, current smoking, current glucocorticoid use, rheumatoid arthritis, secondary osteoporosis and alcohol use ( $>3$ units/day), alone, or by integration of CRFs with BMD hip T-score (FRAX BMD); finally, a FRAX computation was made, adjusted for trabecular bone score (TBS-adj FRAX).

\section{Statistical analysis}

Results are presented as mean values \pm 1 standard deviation (SD). Significance between means was assessed using the Student's $t$-test. Frequencies were compared using chisquared test. Pearson correlation coefficient was used to assess the relationship between continuous variables, in particular between cIMT and biochemical parameters, DXA values, as well as FRAX. Receiver operating characteristic (ROC) curves were designed to identify which of the parameters studied was best suited to predict the cIMT. For each ROC curve, specificity and sensitivity were calculated (95\% confidence interval, CI). The statistical analyses were carried out using the Statistical Package for the Social Sciences software (release 20; SPSS Inc., Chicago, IL, USA). The results were considered significant when the probability value was less than $0.05 \%$ ( $p$ value $<0.05$ ).

\section{Results}

Ten patients were excluded based on the inclusion criteria, among the 100 subjects screened. Considering the 90 patients enrolled, we found 32 patients with a cIMT $\geq 0.9 \mathrm{~mm}$ ( $35 \%$ of the population, group A). There were no differences in anthropometric parameters between group A and those subjects with a cIMT lower than 0.9 (group B), as shown in Table 1. There were no differences in cardiovascular risk factors between group A and group B. Indeed, hypertension was found in approximately $31 \%$ of patients in group A compared to $25 \%$ in group B $(p=0.63)$. Ace-inhibitors were the most frequent drug prescribed, with no difference between the groups. Dyslipidemia was found in 25\% of the subjects in group A and 18\% in group B $(p=0.59)$. Statins were prescribed in $18 \%$ of subjects in group A and $12 \%$ in group B $(p=0.53)$. Approximately $8 \%$ of the patients in group B had both hypertension and dyslipidemia compared to $12 \%$ in group A $(p=0.71)$. Considering other traditional risk factors for atherosclerosis, both the alcohol consumption ( $>3$ units/day) and the current smoking habits did not different between group A and group B (Table 1).

A higher prevalence of osteoporosis was found in group A compared to group B (46.8 vs $22.4 \%, p=0.03$ ) and also a higher number of non-vertebral fractures (15.6 vs $1.72 \%$, $p=0.02$ ), as shown in Tables 1 and 2. Mean values of FRAX $\mathrm{H}$ and $\mathrm{M}$, computed without BMD, did not differ between the two groups (Table 2). However, the mean FRAX values adding femoral neck T-score values, were statistically significantly higher in group A for hip (FRAX BMD H) and for major fracture (FRAX BMD M) compared to group $\mathrm{B}$ (Table 2). Also, mean values of TBS-adj FRAX were significantly higher in group A compared to group B for both hip (TBS-adj FRAX H) and for major fracture (TBS-adj FRAX $\mathrm{M})$. In particular, considering the threshold of 3\% to define a subject at high risk for hip fracture for TBS-adj FRAX H, half of the subjects in group A were above this threshold; the double compared to group B (50 vs $25 \%, p=0.03$ ).

Mean values of biochemical parameters were not statistically different between the two groups (Table 3 ). The

Table 1 Mean \pm SD or percentage of the parameters needed for FRAX computation in the sample divided according to cIMT

\begin{tabular}{lll}
\hline & $\begin{array}{l}\text { cIMT } \geq 0.9 \\
\text { Group A }\end{array}$ & $\begin{array}{l}\text { cIMT }<0.9 \\
\text { Group B }\end{array}$ \\
\hline Age (years) & $61.53 \pm 7.39$ & $61.79 \pm 8.89$ \\
Body mass index $\left(\mathrm{kg} / \mathrm{m}^{2}\right)$ & $24.50 \pm 4.04$ & $24.19 \pm 4.08$ \\
Parental hip fracture & $22 \%$ & $17 \%$ \\
Vertebral fractures & $22 \%$ & $15.5 \%$ \\
Peripheric fracture & $15.6 \%$ & $1.72 \% *$ \\
Glucocorticoid & $0 \%$ & $0 \%$ \\
Rheumatoid arthritis & $0 \%$ & $0 \%$ \\
Secondary osteoporosis & $0 \%$ & $0 \%$ \\
Alcohol $>3$ units/day & $12.5 \%$ & $9 \%$ \\
Current smoking & $31 \%$ & $34 \%$ \\
Trabecular bone score & $1.050 \pm 0.14$ & $1.110 \pm 0.87$ \\
T-score neck & $-1.98 \pm 0.88$ & $-1.47 \pm 0.75^{*}$ \\
\hline
\end{tabular}

$* p<0.05$ 
Table 2 Mean \pm SD or percentage of bone parameters and FRAX in the sample dived according to cIMT

\begin{tabular}{lll}
\hline & $\begin{array}{l}\text { cIMT } \geq 0.9 \\
\text { Group A }\end{array}$ & $\begin{array}{l}\text { cIMT }<0.9 \\
\text { Group B }\end{array}$ \\
\hline Osteoporosis (\%) & 46.8 & $22.4^{*}$ \\
Osteopenia (\%) & 43.7 & 56.8 \\
Normal (\%) & 9.3 & 20.6 \\
T-score L1-L4 & $-2.25 \pm 1.24$ & $-1.67 \pm 1.27^{*}$ \\
T-score total & $-1.63 \pm 0.97$ & $-1.11 \pm 0.87^{*}$ \\
FRAX M (\%) & $6.39 \pm 4.72$ & $6.5 \pm 2.08$ \\
FRAX H (\%) & $1.84 \pm 2.52$ & $2.08 \pm 2.92$ \\
FRAX BMD M (\%) & $8.17 \pm 6.27$ & $5.95 \pm 4.21^{*}$ \\
FRAX BMD H (\%) & $2.86 \pm 3.5$ & $1.51 \pm 2.14^{*}$ \\
TBS-adj FRAX M (\%) & $12.95 \pm 9.6$ & $8.79 \pm 4.89^{* *}$ \\
TBS-adj FRAX H (\%) & $5.36 \pm 6.27$ & $2.39 \pm 2.20^{* *}$ \\
\hline
\end{tabular}

$H$ hip, $M$ major fractures, $T B S-a d j$ trabecular bone score adjusted, $B M D$ bone mineral density; $* p<0.05, * * p<0.001$

Table 3 MeanSD of the principle biochemical parameters of the sample divided according to cIMT

\begin{tabular}{lcc}
\hline & cIMT $\geq 0.9$ & cIMT $<0.9$ \\
& Group A & Group B \\
\hline Creatinine (mg/dl) & $0.76 \pm 0.13$ & $0.82 \pm 0.15$ \\
Calcium (mg/dl) & $9.34 \pm 0.36$ & $9.36 \pm 0.48$ \\
Phosphorus (mg/dl) & $3.65 \pm 0.60$ & $3.73 \pm 3.65$ \\
25(OH)D (ng/ml) & $23.69 \pm 11.61$ & $24.02 \pm 8.19$ \\
PTH (ng/l) & $24.93 \pm 8.73$ & $24.02 \pm 8.19$ \\
BALP (U/l) & $12.17 \pm 4.33$ & $13.08 \pm 5.49$ \\
CTX (ng/ml) & $0.56 \pm 0.27$ & $0.55 \pm 0.26$ \\
RANKL (pmol/l) & $233.5 \pm 144.36$ & $203.76 \pm 162.31$ \\
OPG (pmol/l) & $8.20 \pm 2.55$ & $9.56 \pm 4.09$ \\
RANKL/OPG & $25.8 \pm 21.58$ & $32.41 \pm 25.47$ \\
Interleukin-6 (pg/ml) & $2.0 \pm 2.08$ & $2.11 \pm 1.61$ \\
\hline
\end{tabular}

$25(\mathrm{OH}) \mathrm{D}$ vitamin D, $P T H$ parathyroid hormone, $B A L P$ bone isoenzyme of alkaline phosphatase, CTX C-terminal Telopeptide of collagen type I, $O P G$ osteoprotegerin, $R A N K L$ receptor activator of nuclear factor kappa-B ligand number of patients with serum levels of $25(\mathrm{OH})$ vitamin D less than $20 \mathrm{ng} / \mathrm{ml}$ was higher in group A compared to group $\mathrm{B}$, but this difference was not statistically significant (40 vs $27 \%, p=0.24$ ). Among patients in group A, patients with vitamin $\mathrm{D}<20 \mathrm{ng} / \mathrm{ml}$ had higher mean creatinine values than those with vitamin $\mathrm{D}>20 \mathrm{ng} / \mathrm{ml}(0.85 \pm 0.13$ vs $0.73 \pm 0.09 \mathrm{mg} / \mathrm{dl}, p=0.004$ ).

Values of cIMT were neither associated with age nor with estimated creatine clearance. Values of cIMT were negatively associated with T-score at lumbar spine $(r=-0.25$, $p=0.025)$ neck $(r=-0.28, p=0.006)$ and total hip $(r=-0.27, p=0.012)$. Moreover, values of cIMT were negatively associated with TBS $(r=-0.27, p=0.010)$. A positive association were found between values of cIMT and FRAX BMD M $(r=0.24, p=0.021)$, with FRAX BMD $\mathrm{H}(r=0.25, p=0.016)$, with TBS-adj FRAX M $(r=0.32$, $p=0.002)$ and with TBS-adj FRAX H $(r=0.34, p=0.001)$.

Both FRAX M and FRAX H were negatively associated with estimated creatine clearance $(r=-0.28, p=0.03$; $r=-0.29, p=0.02$, respectively).

Receiver operating characteristic (ROC) curves showed a high specificity for both neck and total hip T-score value (with a threshold of $-2.5 \mathrm{SD}$ ), but showed a low sensibility in identifying patients with cIMT $\geq 0.9 \mathrm{~mm}$ (Table 4). The ROC curve of FRAX H and M, of FRAX BMD M showed that none of these parameters was able to identify subjects with subclinical atherosclerosis (all $p>0.05$, Table 4). Also, TBS values were not able to identify subjects with subclinical atherosclerosis $(p>0.05)$. The best ROC curve was for lumbar spine T-score. Considering a T-score threshold $-2.5 \mathrm{SD}$, it was possible to identify subjects with a cIMT $\geq 0.9 \mathrm{~mm}$, with a sensibility of $50 \%$ and with a specificity of 76\% (area under the curve, AUC 0.64; 95\% confidence interval (CI) 0.52-0.76, $p=0.02$ ). Similarly, TBS-adj FRAX $\mathrm{H}$ had a sensibility of $50 \%$, and a specificity of $72 \%$ (AUC 0.64; 95\% CI 0.52-0.77, $p=0.01$, with a threshold of $3 \%)$. Furthermore, TBS-adj FRAX M had a low sensibility and a high specificity compared to TBS-adj FRAX $\mathrm{H}$, as shown in Table 4.
Table 4 Area under the curve (AUC), sensibility and specificity for ROC curves that identify subjects with cIMT $\geq 0.9 \mathrm{~mm}(p$ value $<0.05)$

\begin{tabular}{lllll}
\hline & Sensibility $(\%)$ & Specificity $(\%)$ & AUC $(95 \% \mathrm{CI})$ & $p$ value \\
\hline T- score lumbar spine & 50 & 76 & $0.64(0.52-0.76)$ & 0.02 \\
T- score femoral neck & 40.6 & 89.6 & $0.67(0.54-0.79$ & 0.007 \\
T- score total hip & 23.3 & 92.8 & $0.65(0.53-0.78)$ & 0.01 \\
FRAX BMD H & 31.2 & 84 & $0.63(0.51-0.76)$ & 0.03 \\
TBS adj-FRAX BMD H & 50 & 72 & $0.64(0.52-77)$ & 0.01 \\
TBS adj-FRAX BMD M & 15.6 & 94.8 & $0.63(0.50-0.75)$ & 0.04 \\
\hline
\end{tabular}

For T-score values, the threshold was set at -2.5

For FRAX BMD $\mathrm{H}$ and TBS adj-FRAX $\mathrm{H}$, the threshold was set at 3\%

For TBS adj-FRAX BMD M, the threshold was set at $20 \%$ 
In the sample as a whole, values of cIMT were not statistically significantly associated with the biochemical parameters measured. However, both osteoprotegerin and interleukin-6 positively correlated with age $(r=0.23, p=0.045$; $r=0.27, p=0.019$, respectively), while the RANKL/OPG ratio correlated negatively $(r=-0.24, p=0.04)$. Interleukin-6 positively correlated with FRAX M $(r=0.28, p=0.01)$ and FRAX H $(r=0.23, p=0.04)$, FRAX BMD M $(r=0.25$, $p=0.002)$ and FRAX BMD H $(r=0.23, p=0.04)$ and TBSadj FRAX M $(r=0.23, p=0.04)$.

\section{Discussion}

To the best of our knowledge, we have shown, for the first time, that the FRAX algorithm (without BMD) does not identify subclinical atherosclerosis in postmenopausal women without known cardiovascular diseases. However, when adding BMD values we observed a significant capability in identifying subclinical atherosclerosis. Moreover, TBS-adj FRAX $\mathrm{H}$ has a similar ability in identifying subclinical atherosclerosis comparted to T-score lumbar spine alone. The risk of developing atherosclerosis increases, with advancing age; however, in our population we have not observed an association between age and cIMT, probably because the majority of the population has a normal value of cIMT. Indeed, our results showed that DXA values are independently associated with cIMT, because adding all of the clinical risk factors such as age, BMI, etc. included in FRAX did not increase the ability to identify subclinical atherosclerosis.

Although both lumbar spine T-score and TBS-adj FRAX $\mathrm{H}$ do not have a high sensibility in identifying subclinical atherosclerosis, they do have a good specificity. The best specificity above $90 \%$ was for hip T-score and TBS-adj FRAX BMD H at the expense of sensibility. This result is in line with previous literature that showed significant association between neck T-score in postmenopausal women and cIMT values [8]. The only study that showed that FRAX, without BMD, was associated with brachial IMT included patients at high risk of cardiovascular disease. In that study, approximately a third of the population involved men and women with diabetes mellitus, $70 \%$ with hypertension, and more than $30 \%$ with a history of previous stroke or coronary artery diseases [25]. This population was very different from the population enrolled in our study, where previous cardiovascular disease and diabetes were exclusion criteria.

For the first time, we also included TBS in the FRAX computation, for detecting subclinical atherosclerosis. It should be noted that in our sample TBS correlates with cIMT but TBS was not able to identify patients with cIMT $\geq 0.9 \mathrm{~mm}$. However, in particular patients, such as those affected by diabetes mellitus type 2 , TBS values were better associated with cIMT than BMD [32]. In diabetes mellitus, TBS has been found to be lower compared to BMD values, indicating a poor bone quality [32]. However, patients enrolled in our study were not affected by secondary osteoporosis, where TBS might be a more sensitive parameter of bone health.

We found a high percentage of patients with $25(\mathrm{OH}) \mathrm{D}$ lower than $20 \mathrm{ng} / \mathrm{ml}$ in the group with subclinical atherosclerosis, which is not surprisingly due to the frequent hypovitaminosis D in postmenopausal women in this age group [33]; this finding was not statistically significant, even though previous studies have indicated an association between low vitamin D levels and atherosclerosis [34]. Low vitamin D is associated with higher serum PTH levels, as has been found in a previous study in subjects with a higher cIMT [35]. However, in our study, we did not find a significant difference in the PTH values between group A and B.

Our study is subject to a few limitations. We did not measure serum glucose levels, considering that an interplay between glucose metabolism and bone, as well as atherosclerosis, is well documented [36, 38]. However, diabetes was one of the exclusion criteria, but because we did not measure serum glucose it is not possible to exclude that we also enrolled patients with fasting glucose and/or impaired glucose tolerance. Lipids were not biochemically assessed, although it should be noted that we did not find differences between patients with or without carotid subclinical atherosclerosis, when a previous diagnosis of dyslipidemia were specifically asked to patients. Adipokines were also not measured, which have been shown to be associated with both bone metabolism and atherosclerosis [39, 40]. Moreover, we only studied women. A recent study, conducted mainly in males in Taiwan, showed a correlation between the coronary artery calcification score and the FRAX with BMD. However, they enrolled a different population compared to that of our study. In fact, $11.1 \%$ were diabetic and $17.3 \%$ had risk factors of secondary osteoporosis [41].

In conclusion, our study found that FRAX without BMD was not able to identify subclinical carotid atherosclerosis; the presence of lumbar spine osteoporosis should suggest subclinical carotid atherosclerosis in a population of postmenopausal women without a previous diagnosis of cardiovascular diseases, independently of an age effect. Although TBS adj-FRAX $H$ showed very similar performance compared to lumbar spine T-score for the identification of subjects with subclinical carotid atherosclerosis, it does not provide additional information compared to using lumbar spine T-score alone.

Our study highlights the interplay between bone and vascular system beyond common identifiable clinical risk factors. The common pathophysiological mechanism, that independently of clinical risk factors underlie this association, should be better evaluated. 
Funding Open Access funding provided by Università degli Studi di Roma La Sapienza. The study was not supported by any fund or grant.

\section{Compliance with ethical standards}

Conflict of interest All authors have no conflicts of interest.

Ethics approval The study was performed in accordance with ethical standard of the institutional research committee and with Helsinki declaration and its later amendments.

Informed consent Informed consent was obtained from all participants.

Open Access This article is licensed under a Creative Commons Attribution 4.0 International License, which permits use, sharing, adaptation, distribution and reproduction in any medium or format, as long as you give appropriate credit to the original author(s) and the source, provide a link to the Creative Commons licence, and indicate if changes were made. The images or other third party material in this article are included in the article's Creative Commons licence, unless indicated otherwise in a credit line to the material. If material is not included in the article's Creative Commons licence and your intended use is not permitted by statutory regulation or exceeds the permitted use, you will need to obtain permission directly from the copyright holder. To view a copy of this licence, visit http://creativecommons.org/licenses/by/4.0/.

\section{References}

1. En Uyl D, Nurmohamed M, van Tuyl L, Raterman H, Lems W (2011) (Sub)clinical cardiovascular disease is associated with increased bone loss and fracture risk; a systematic review of the association between cardiovascular disease and osteoporosis. Arthritis Res Ther 13:R5. https://doi.org/10.1186/ar3224

2. Veronese N, Stubbs B, Crepaldi G, Solmi M, Cooper C, Harvey NC, Reginster JY, Rizzoli R, Civitelli R, Schofield P, Maggi S, Lamb SE (2017) Relationship between low bone mineral density and fractures with incident cardiovascular disease: a systematic review and meta-analysis. J Bone Miner Res 32(5):1126-1135. https://doi.org/10.1002/jbmr.3089

3. Ye C, Xu M, Wang S, Jiang S, Chen X, Zhou X, He R (2016) Decreased bone mineral density is an independent predictor for the development of atherosclerosis: a systematic review and metaanalysis. PLoS ONE 11:e0154740. https://doi.org/10.1371/journ al.pone. 0154740

4. Zhang Y, Fang X, Hua Y, Tang Z, Guan S, Wu X, Liu H, Liu B, Wang C, Zhang Z, Gu X, Hou C, Liu C (2018) Carotid artery plaques, carotid intima-media thickness, and risk of cardiovascular events and all-cause death in older adults: a 5-year prospective, community-based study. Angiology 69:120-129. https://doi. org/10.1177/0003319717716842

5. Tamaki J, Iki M, Hirano Y, Sato Y, Kajita E, Kagamimori S, Kagawa Y, Yoneshima H (2009) Low bone mass is associated with carotid atherosclerosis in postmenopausal women: the Japanese Population-based Osteoporosis (JPOS) Cohort Study. Osteoporos Int 20:53-60. https://doi.org/10.1007/s00198-008-0633-z

6. Värri M, Tuomainen TP, Honkanen R, Rikkonen T, Niskanen L, Kröger H, Tuppurainen MT (2014) Carotid intima-media thickness and calcification in relation to bone mineral density in postmenopausal women-the OSTPRE-BBA study. Maturitas 78:304-309. https://doi.org/10.1016/j.maturitas.2014.05.017
7. Shaffer JR, Kammerer CM, Rainwater DL, O'Leary DH, Bruder JM, Bauer RL, Mitchell BD (2007) Decreased bone mineral density is correlated with increased subclinical atherosclerosis in older, but not younger, Mexican American women and men:the San Antonio Family Osteoporosis Study. Calcif Tissue Int 81:430-441. https://doi.org/10.1007/s00223-007-9079-0

8. Campos-Staffico AM, Freitas WM, Carvalho LSF, Coelho-Filho OR, Nadruz W Jr, Oliveira RB, Sposito AC, Brasilia Study on Healthy Aging and Brasilia Heart Study (2020) Lower bone mass is associated with subclinical atherosclerosis, endothelial dysfunction and carotid thickness in the very elderly. Atherosclerosis 292:70-74. https://doi.org/10.1016/j.atherosclerosis.2019.11.007

9. Rossi M, Battafarano G, Pepe J, Minisola S, Del Fattore A (2019) The endocrine function of osteocalcin regulated by bone resorption: a lesson from reduced and increased bone mass diseases. Int J Mol Sci 20:E4502. https://doi.org/10.3390/ijms20184502

10. Morisawa T, Nakagomi A, Kohashi K, Kosugi M, Kusama Y, Atarashi H, Shimizu W (2015) Osteoprotegerin is associated with endothelial function and predicts early carotid atherosclerosis in patients with coronary artery disease. Inter Heart J 56:605-612. https://doi.org/10.1536/ihj.15-150

11. Liu W, Zhang X (2015) Receptor activator of nuclear factor- $\kappa B$ ligand (RANKL)/RANK/osteoprotegerin system in bone and other tissues (review). Mol Med Rep 11:3212-3218. https://doi. org/10.3892/mmr.2015.3152

12. Minisola S, Cipriani C, Cilli M, Scillitani A, Pepe J (2015) A reappraisal of vitamin D effect on non-skeletal targets and mortality. J Endocrinol Invest 38:1239-1241. https://doi.org/10.1007/s4061 8-015-0387-3

13. Saponaro F, Marcocci C, Zucchi R (2019) Vitamin D status and cardiovascular outcome. J Endocrinol Invest 42:1285-1290. https ://doi.org/10.1007/s40618-019-01057-y

14. Cellini M, Piccini S, Ferrante G, Carrone F, Olivetti R, Cicorella N, Aroldi M, Pini D, Centanni M, Lania AG, Mazziotti G (2020) Secondary hyperparathyroidism and thoracic vertebral fractures in heart failure middle-aged patients: a 3-year prospective study. J Endocrinol Invest 43:1561-1569. https://doi.org/10.1007/s4061 8-020-01237-1

15. Cainzos-Achirica M, Enjuanes C, Greenland P, McEvoy JW, Cushman M, Dardari Z, Nasir K, Budoff MJ, Al-Mallah MH, Yeboah J, Miedema MD, Blumenthal RS, Comin-Colet J, Blaha MJ (2018) The prognostic value of interleukin 6 in multiple chronic diseases and all-cause death: the Multi-Ethnic Study of Atherosclerosis (MESA). Atherosclerosis 278:217-225. https:// doi.org/10.1016/j.atherosclerosis.2018.09.034

16. Barbour KE, Lui LY, Ensrud KE, Hillier TA, LeBlanc ES, Ing SW, Hochberg MC, Cauley JA, Study of Osteoporotic Fractures (SOF) Research Group (2014) Inflammatory markers and risk of hip fracture in older white women: the study of osteoporotic fractures. J Bone Miner Res 29:2057-2064. https://doi.org/10.1002/ jbmr.2245

17. Spinelli R, Parrillo L, Longo M, Florese P, Desiderio A, Zatterale F, Miele C, Alexander Raciti G, Beguinot F (2020) Molecular basis of ageing in chronic metabolic diseases. J Endocrinol Invest 43:1373-1389. https://doi.org/10.1007/s40618-020-01255-Z

18. Liu J, Curtis EM, Cooper C, Harvey NC (2019) State of the art in osteoporosis risk assessment and treatment. J Endocrinol Invest 42:1149-1164. https://doi.org/10.1007/s40618-020-01255-Z

19. Cipriani C, Pepe J, Bertoldo F, Bianchi G, Cantatore FP, Corrado A, Di Stefano M, Frediani B, Gatti D, Giustina A, Porcelli T, Isaia G, Rossini M, Nieddu L, Minisola S, Girasole G, Pedrazzoni M (2018) The epidemiology of osteoporosis in Italian postmenopausal women according to the National Bone Health Alliance (NBHA) diagnostic criteria: a multicenter cohort study. J Endocrinol Invest 41(4):431-438. https://doi.org/10.1007/s4061 8-017-0761-4 
20. Szekanecz Z, Raterman HG, Pethő Z, Lems WF (2019) Common mechanisms and holistic care in atherosclerosis and osteoporosis. Arthritis Res Ther 21:15. https://doi.org/10.1186/s1307 5-018-1805-7

21. Dessein PH, Semb AG, González-Gay MA, Popa CD (2015) Enhanced cardiovascular risk in rheumatoid arthritis: elucidation, assessment, and management. Biomed Res Int 2015:850671. https ://doi.org/10.1155/2015/850671

22. Hamada M, Kajita E, Tamaki J, Kouda K, Sato Y, Tachiki T, Yura A, Kamiya K, Nitta A, Kagamimori S, Iki M (2020) Decreased bone mineral density and osteoporotic fractures are associated with the development of echogenic plaques in the carotid arteries over a 10-year follow-up period: the Japanese Population-based Osteoporosis (JPOS) Cohort Study. Maturitas 131:40-47. https:// doi.org/10.1016/j.maturitas.2019.10.010

23. Kanis JA, Johnell O, Oden A, Johansson H, McCloskey E (2008) FRAX and the assessment of fracture probability in men and women from the UK. Osteoporos Int 19:385-397. https://doi. org/10.1007/s00198-007-0543-5

24. Warzecha M, Czerwiński E, Amarowicz J, Berwecka M (2018) Trabecular Bone Score (TBS) in clinical practicereview. Ortop Traumatol Rehabil 20:347-359. https://doi. org/10.5604/01.3001.0012.7281

25. Kajikawa M, Oda N, Kishimoto S, Maruhashi T, Iwamoto $\mathrm{Y}$, Iwamoto A, Matsui S, Aibara Y, Mohamad Yusoff FB, Hidaka T, Kihara Y, Chayama K, Goto C, Noma K, Nakashima A, Taguchi A, Higashi Y (2017) Increasing risk of osteoporotic fracture is associated with vascular dysfunction and abnormal vascular structure in both men and women. Circ J 81:862-869. https://doi. org/10.1253/circj.CJ-16-1236

26. Expert Panel on Detection, Evaluation, and Treatment of High Blood Cholesterol in Adults (2019) Executive Summary of the Third Report of the National Cholesterol Education Program (NCEP) Expert Panel on Detection, Evaluation, and Treatment of High Blood Cholesterol in Adults (Adult Treatment Panel III). JAMA 285:2486-2497. https://doi.org/10.1001/jama.285.19.2486

27. Pepe J, Diacinti D, Fratini E, Nofroni I, D’Angelo A, Pilotto R, Savoriti C, Colangelo L, Raimo O, Cilli M, Cipriani C, Minisola $S$ (2016) High prevalence of abdominal aortic calcification in patients with primary hyperparathyroidism as evaluated by Kauppila score. Eur J Endocrinol 175:95-100. https://doi.org/10.1530/ EJE-15-1152

28. Paglia F, Dionisi S, De Geronimo S, Rosso R, Romagnoli E, Raejntroph N, Ragno A, Celi M, Pepe J, D'erasmo E, Minisola S (2001) Biomarkers of bone turnover after a short period of steroid therapy in elderly men. Clin Chem 47:1314-1316. https://doi. org/10.1093/clinchem/47.7.1314

29. Pedrazzoni M, Girasole G, Bertoldo F, Bianchi G, Cepollaro C, Del Puente A, Giannini S, Gonnelli S, Maggio D, Marcocci C, Minisola S, Palummeri E, Rossini M, Sartori L, Sinigaglia L (2003) Definition of a population-specific DXA reference standard in Italian women: the Densitometric Italian Normative Study (DINS). Osteoporos Int 14(12):978-982. https://doi.org/10.1007/ s00198-003-1521-1

30. Gottdiener JS, Bednarz J, Devereux R, Gardin J, Klein A, Manning WJ, Morehead A, Kitzman D, Oh J, Quinones M, Schiller NB, Stein JH, Weissman NJ, American Society of Echocardiography
(2004) American Society of Echocardiography recommendations for use of echocardiography in clinical trials. J Am Soc Echocardiograp 17:1086-1119. https://doi.org/10.1016/j.echo.2004.07.013

31. FRAX: WHO fracture risk assessment tool. http://www.shef. ac.uk/frax/.

32. Caffarelli C, Giambelluca A, Ghini V, Francolini V, Pitinca MDT, Nuti R, Gonnelli S (2017) In type-2 diabetes subjects trabecular bone score is better associated with carotid intima-media thickness than bmd. Calcif Tissue Int 101:404-411. https://doi.org/10.1007/ s00223-017-0297-9

33. Minisola S, Colangelo L, Pepe J, Occhiuto M, Piazzolla V, Renella M, Biamonte F, Sonato C, Cilli M, Cipriani C (2020) Vitamin D screening. J Endocrinol Invest 43:1047-1051. https://doi. org/10.1007/s40618-020-01220-w

34. Wang Y, Zhang H (2017) Serum 25-hydroxyvitamin d3 levels are associated with carotid intima-media thickness and carotid atherosclerotic plaque in type 2 diabetic patients. J Diabetes Res 2017:3510275. https://doi.org/10.1155/2017/3510275

35. Lee YH, Kweon SS, Choi JS, Nam HS, Park KS, Choi SW, Ryu SY, Oh SH, Shin MH (2017) Association of serum vitamin D and parathyroid hormone with subclinical atherosclerotic phenotypes: the Dong-gu Study. PLoS ONE 12:e0186421. https://doi. org/10.1371/journal.pone.0186421

36. Cipriani C, Colangelo L, Santori R, Renella M, Mastrantonio M, Minisola S, Pepe J (2020) The interplay between bone and glucose metabolism. Front Endocrinol 11:122. https://doi.org/10.3389/ fendo.2020.00122

37. Giugliano D, Chiodini P, Maiorino MI, Bellastella G, Esposito K (2019) Cardiovascular outcome trials and major cardiovascular events: does glucose matter? A systematic review with meta-analysis. J Endocrinol Invest 42:1165-1169. https://doi.org/10.1007/ s40618-019-01047-0

38. Morales Gurrola G, Simental Mendía LE, Castellanos Juárez FX, Salas Pacheco JM, Guerrero Romero F (2020) The triglycerides and glucose index is associated with cardiovascular risk factors in metabolically obese normal weight subjects. J Endocrinol Invest 43:995-1000. Doi:https://doi.org/10.1007/s40618-020-01184-x

39. Barchetta I, Cimini FA, Ciccarelli G, Baroni MG, Cavallo MG (2019) Sick fat: the good and the bad of old and new circulating markers of adipose tissue inflammation. J Endocrinol Invest 42:1257-1272. https://doi.org/10.1007/s40618-019-01052-3

40. Pepe J, Cipriani C, Cilli M, Colangelo L, Minisola S (2016) Adipokines and bone metabolism: an interplay to untangle. J Endocrinol Invest 39:1359-1361. https://doi.org/10.1007/s4061 8-016-0549-y

41. Chuang TL, Li YD, Hsiao FT, Chuang MH, Wang YF (2017) FRAX ${ }^{\circledR}$ fracture risks are associated with coronary artery calcification score. Dis Markers 2017:1592598. https://doi. org/10.1155/2017/1592598

Publisher's Note Springer Nature remains neutral with regard to jurisdictional claims in published maps and institutional affiliations. 